\title{
PREDICTING CONSUMER RESPONSE TO NEW HOUSING: A STATED CHOICE EXPERIMENT
}

Eric Molin, Harmen Oppewal and Harry Timmermans

ABSTRACT This paper discusses problems typical of eliciting housing preference. It will be argued that stated preference and choice models are potentially powerful in eliciting consumer housing preferences. This approach is illustrated in an example of new housing construction in Meerhoven. The design of the stated choice experiment is outlined and the estimated part-worth utilities of the attributes are presented. Furthermore, choices for houses in low- and high-density environments are predicted and it is examined how much more households are willing to pay for low-density housing.

\section{Introduction}

Over the past decades, the housing market in many industrialized countries has moved away from a heavily government-regulated industry to an increasingly market-orientated industry. This trend reflects a retreat of government in providing social facilities. Other non-profit or profit organizations in the market place have been given greater autonomy and have been empowered to make their own decisions. Consequently, housing associations, for example, have felt an increased need to build houses that reflect the needs and preferences of their target market. It has led to an upsurge of market research activities to predict consumer response to new housing products. Newly constructed houses should satisfy the needs and preferences of households for which these houses are intended. To the extent that housing associations are successful in attaining these goals, risks are reduced.

From an academic point of view, this increased market orientation raises the question how housing preferences can be measured. Realizing that no method is necessarily error-free, it seems critical to identify the specific assumptions underlying any particular method and assess to what extent these assumptions apply to any specific problem. The purpose of the present paper is to summarize some of the methodological discussion related to alternative ways of eliciting consumer preferences. It will be argued that stated choice models are especially valuable when

Neth. J. of Housing and the Built Environment, Vol. 11 (1996) No. 3. 
consumer reaction to completely new housing types, for which historical data are lacking, is sought for. The method will be illustrated using a project in Meerhoven, Eindhoven's VINEX locations, as an example.

The organization of the paper strictly follows these goals and objectives. We will first summarize the advantages and disadvantages of alternative methods of eliciting consumer preferences and discuss how these relate to housing. Then, we will outline the design of a stated choice experiment and illustrate its use in predicting consumer response to new housing construction. Finally, we will draw some conclusions to finish the article.

\section{Eliciting consumer preferences}

The problem of how to measure consumer preferences has plagued numerous disciplines over the last decades. Many different theories, models and methods have been advocated, but unfortunately systematic comparative research is still largely lacking. Consequently, any discussion of the strengths and weaknesses of alternative means of eliciting preferences necessarily has to be largely based on theoretical positions and logic.

Some scholars seem to believe that it is only in the act of actual choice that individuals can reveal their preferences. That standpoint is based on the premise that some rationality is underlying consumer behaviour: choices are assumed to reflect preferences. If an individual is observed to choose alternative A over B, C, D etc., it is assumed that the preference for A exceeds the individual's preference for any other choice alternative in his choice set. Realizing that preferences may be stochastic, repeated measurements would be required to elicit the preference functions, but the same basic principle would apply. In order to determine which characteristics or attributes of the choice alternatives influence preferences, one typically specifies a model that meets some basic statistical requirements such as the fact that the problem involves discrete data. Essentially, however, these revealed preference models interpret observed consumer choice as a manifestation of utilitymaximizing behaviour (highest preference). The implied utility or preference function is identified by relating observed choices to a set of attributes describing the choice alternatives using an appropriate statistical model. This procedure will depict the strength of the relationship between observed choice and the selected attributes of the choice alternatives.

Now, if we forget that preferences may be conditional and that individuals may be involved in variety-seeking behaviour, it seems reasonable to assume that actual choice reflects average preferences if an individual can freely express his preferences. If we, for example, observe that a particular percentage of the market lives in apartment buildings, can we conclude that this percentage of consumers prefers apartments to other types of dwellings? Of course, the answer is NO: in addition to consumer preferences, observations of residential behaviour reflect the supply-demand conditions of the market. If individuals cannot find the houses they prefer because of insufficient supply, they either have to decide to postpone their 
housing decision or choose an alternative of lower preference. In any case, any predictions based on observed choices or market share would be biased and misleading, unless the supply-demand conditions and the (dis)equilibrium of the systems would be left unchanged. As these conditions are unlikely to be met in many applied contexts, the relevancy of revealed preference models to predict consumer response to new housing tends to be very limited.

In addition to this fundamental shortcoming, some other characteristics of this approach further reduce their potential for predicting consumer response to new housing construction. First, the attributes of housing alternatives in real markets may show high correlations; for example, bigger houses are typically more expensive. As the correlations between the explanatory variables increase, bias tends to increase up to near-multicollinearity. In that event, it is well known that the estimation can result in misleading estimates, including wrong signs. Consequently, the parameter estimates of revealed preference models in housing applications reflect not only market (dis)equilibriums but also the correlational structure of the selected attributes, as observed in the market place, and hence are highly suspect.

Secondly, because one relies on observed choice, it is not readily evident how revealed preference models should be used if new housing construction deviates from earlier construction. It is well established that scholars have no problem applying any model for forecasting if the values of the explanatory variables fall within the domain or range of observations that has been used to estimate the model. In fact, this is the quintessence of model-building: one examines the data to see if some systematic relationship can be found between a dependent variable and a set of explanatory variables; one then tries to capture this relationship, if present, in terms of some mathematical or logical expression; that equation is then used for forecasting under the assumption that the relationship is invariant over time. However, one necessarily has to make more rigorous assumptions if the values of the explanatory variables are beyond the domain of observations. In this case, lacking empirical evidence that the assumed model is valid, one has to rely on the untestable assumption that the model generalizes beyond one's observations. Although this reasoning is well accepted for single explanatory variables, in our opinion it also holds for specific combinations of values of the explanatory variables that have not been observed before. Note that, as we have argued earlier, the researcher has no control over the data. Consequently, there are likely to be many sub-pairs (combinations of values of explanatory variables) in the multi-dimensional space that spans the range of the explanatory variables included in the model for which no empirical observations are available. This will not only bias the model's estimates but also influence the kind of assumptions one has to make to use the model for forecasting. In particular, one would have to make assumptions about the values of explanatory variables for which observations are missing. In some cases, this may be a realistic assumption. The more the new housing construction deviates from the past for which data are available, the less realistic this assumption becomes, up to the point that the historical data become virtually irrelevant. In that extreme case, revealed preference models cannot be developed, simply because historical data are non-existent. 
Given all these potential problems, another group of researchers seems to believe that one can simply ask respondents anything to elicit their preferences. Although this position is appealing, unfortunately this task is much mere complicated than one might expect. First, it is based on the assumption that individuals are actually able to articulate their preferences. In reality, they may be indifferent, or their preferences may depend on particular conditions, implying that the measurement task should be sufficiently complex to represent these circumstances. It is highly unlikely that one simple question will produce the required information.

Secondly, individuals are supposed to tell the truth. Measurements may be influenced by response effects. For example, there is a rich accumulation of empirical evidence that attitudes are not systematically related to behaviour. Likewise, Tversky's theory suggests that the framing of questions leads to different response patterns (Tversky, 1972). Although these are serious considerations, we feel they are potentially of lesser concern in housing applications, as this is a decision that is less influenced by considerations of social desirability and risky decision-making. Nevertheless, the validity of the responses is a concern, and the ideal method should incorporate elements to test the validity of the measurements.

Thirdly, the validity of one's measurements will be influenced by the degree to which the process through which the information is obtained reflects the essentials of the actual decision-making process. In our opinion, this is perhaps the most serious problem. Conventional wisdom in survey and questionnaire design is to keep the questions as simple as possible. However, when used uncritically, this guideline may imply that we are measuring whatever we are measuring very precisely, but we may be precisely measuring the wrong thing! Questions in survey research will either implicitly or explicitly be derived from some conceptual framework that is supposed to represent the behaviour of interest. To the extent that this framework (and hence the questions, form of prompting, or the measurement procedure as such) fails to capture the essential characteristics of whatever one is trying to measure, it is doubtful that valid measurements can be obtained. Given these considerations, it should be evident that measurements of consumer preferences are necessarily dependent upon the conceptual considerations that are imposed. Consequently, it is of utmost importance that the measurement procedure incorporates the elements necessary to test the validity of the assumptions underlying the measurements. Data should be fallible. Rather than insisting on a set of simple questions that cannot be tested, a better way of eliciting preferences is to develop a measurement procedure which involves different sets of interrelated data that allows one to test various aspects of validity. To the extent that the conceptual considerations that have driven the data collection are supported by the data, there is greater reason to believe one has more valid and more reliable data on consumer preferences.

Keeping these considerations in mind, some scholars have assumed that when forming preferences, individuals first identify the attributes of the choice alternatives on the basis of which they form their preferences. They value each of these attributes and then arrive at an overall preference by combining their valuations of attributes, typically according to a weighted linear function, where the weights depict the relative importance of the attributes. In terms of measurement procedure, 
the so-called compositional stated preference models assume that preference can be elicited by asking respondents to express their evaluation or satisfaction separately for a set of influential housing attributes and to measure, also explicitly and separately, the relative importance of the weights of these attributes. Overall preferences can then be easily constructed by combining these measurements according to, for example, a linear-additive preference function. Obviously, this is a simple and straightforward measurement procedure. Whether it is also valid remains debatable. The approach implicitly assumes that respondents are capable of expressing their evaluation of separate housing attributes, not knowing what to assume about the values of the remaining attributes influencing their preference. Hence, one is actually assuming that respondents can express an average evaluation with a single direct question. That is, one assumes that respondents can, for instance, attach some numerical evaluation score to a price of a house without knowing what the house has to offer in terms of number of rooms, garden, location etc. Likewise, the approach assumes that individuals are capable of expressing the relative importance of these attributes in terms of simple, direct questions. Obviously, both are very strong assumptions, and in fact there is now a consistent accumulation of empirical evidence which suggests that the validity of this approach is rather weak. Compositional stated preference models have attempted to elicit consumer preference by breaking down the measurement procedure into a series of direct, simple questions. However, empirical evidence suggests this has been an over-simplification that fails to capture the essence of the housing choice process and seems to be too demanding after all for respondents to articulate their preferences. What is needed then is an approach that better reflects the choice process and that represents a more systematic approach in eliciting consumer preferences. Stated preference and choice models (e.g., Timmermans, 1984; Louviere, 1988; and Louviere and Timmermans, 1990a) have more to offer in these respects.

\section{Stated preference and choice models}

It has been argued that an important feature of any measurement procedure is that it is derived from the conceptual framework that is supposed to explain the behaviour of interest and that allows one to test, and hence reject, the assumptions made to formally represent this behaviour. Thus, let us first summarize the conceptual model underlying stated preference and choice models.

Let us first assume that any choice alternative such as a house or residential environment can be described as a multi-dimensional profile of attributes. The profile describes the position of a particular choice alternative along multiple dimensions such as price, tenure, location, density, number of bedrooms, etc. Individuals are assumed to derive some utility from each of these attributes. This utility function can take on any form: it may be monotonically increasing or decreasing with attribute values; it may also be dependent on specific attribute levels. To arrive at a preference scale, which positions the choice alternatives in terms of overall preference, individuals are assumed to trade-off their attribute 
utilities according to some combination rule. Although complex structures can be assumed (e.g., Timmermans and van Noortwijk, 1995), often a compensatory decision-making process is assumed. In that case, one assumes that a low value of one attribute can, at least partially, be compensated by higher values on one or more of the remaining attributes. It can be formally represented by a linear-additive preference function. Moreover, when interested in choices as opposed to preferences, individuals are supposed to choose the alternative which yields the highest preference. Assuming stochastic preference, to derive a choice model one has to make additional assumptions regarding the distributions of the stochastic elements of the preference function. A simple model that can be derived from stochastic preference and the principle of utility-maximizing behaviour is the multinominal logit model.

Now, it is believed that a valid measurement procedure should (i) incorporate mechanisms that reflect the assumed trade-off nature of the decision-making process, (ii) allow one to critically test the assumed compensatory decision-making process as reflected in the linear-additive preference function, and (iii) if relevant, allow one to critically test the assumed utility-maximizing behaviour as reflected in the multinominal logit model. In order to satisfy these requirements, stated preference and choice models do not rely on direct, simple questions but take respondents through a series of trade-offs. When making these trade-offs, respondents are implicitly providing information about their utilities and implied attribute importance. The problem then becomes how these series of trade-offs should be optimally designed such as to (i) satisfy the necessary and sufficient conditions to test the assumed preference and/or choice models and (ii) avoid respondent fatigue, response effects, etc., thereby increasing the reliability of the measurements.

Stated preference and choice models typically design these trade-offs according to the principles underlying the design of statistical experiments. The basic design problem is how to develop an experiment with a minimum number of profiles or choice sets that allows one to test the assumptions one has made regarding the preference function and the choice models. For example, evidence supporting an assumed additive preference function would be obtained if the design permits one to test the significance of interaction effects and the test would indicate none of the estimated interactions to be significant. Likewise, the validity of the multinominal logit model would be supported if the data would demonstrate that the utility of a particular choice alternative is not significantly influenced by the existence and/or attribute levels of any other alternative in this choice set. Although the design of such experiments is often difficult, stated preference and choice models have the potential advantage that one does not have to assume that the measurements and the model of preference function are valid, but that one can actually critically test the validity of the model. This is not to say that these tests are always applied to the extent possible in applications of stated preference and choice models. Especially in many applied projects, one often only tests whether the model is capable of reproducing the stated choice or preference data. 


\section{$4 \quad$ An illustration}

\subsection{Context}

The use of stated preference and choice models of housing choice will be illustrated using Meerhoven as an example. The planning problem was to design a new neighbourhood that would contain new housing for mainly middle-class tenants. Current Dutch policy encourages cities and city regions to concentrate new housing in an attempt to safeguard rural areas. Therefore, housing in Meerhoven must be built at a higher density than what is considered normal in the Eindhoven region. As most housing is built without financial aid from the central government, the costs will be above average. Therefore, new tenants must come from middle and higher income groups. The general picture of the residential preferences of these groups is that they are not really willing to move because they already live in good dwellings; if they are willing to move, they are looking for spacious housing. Therefore, the local government likes to have more insight in the housing choice process of the target group, especially with respect to higher density. To this effect, a conjoint choice experiment is developed, in which the housing profiles include housing and house-environmental attributes.

\subsection{The experiment}

The first step in building a stated preference model is to identify the attributes of interest. On the one hand, this involves an attempt to select those attributes that influence the consumer choice behaviour under investigation. On the other hand, it also involves a policy analysis to identify the "design" or "marketing" attributes that are relevant to policy-makers, urban designers, or marketers, even though these attributes may not be necessarily relevant to consumers. Often, attributes are elicited by small-scale qualitative research, decision nets or tables or factor listings being good examples of such research endeavours.

In the present study, attributes were identified on the basis of our previous research efforts (e.g., Timmermans, 1989; Louviere and Timmermans, 1990b; Molin et al., 1995) and feedback from experts. The list of selected attributes and their levels is portrayed in Figure 1.

Once the attributes and their levels have been identified, an experimental design that systematically varies these attributes across trade-off situations should be constructed. Ideally, the design should allow one to (i) obtain unbiased estimates of the parameters of the preference function, (ii) test the assumed structure of the preference function, (iii) test the assumed form of the choice model, and (iv) avoid response effects and the like. Moreover, it should also be feasible in terms of respondent demand, costs, and administration. Finally, in order to increase the validity, the choice situation in the experiment must resemble choice situations in real housing markets as much as possible. 
Figure 1 Selected attributes and their levels

\begin{tabular}{|c|c|c|}
\hline $\begin{array}{l}\text { tenure } \\
\text { - rent } \\
\text { - own }\end{array}$ & $\begin{array}{l}\text { size living room } \\
-20 \mathrm{~m}^{2} \\
-30 \mathrm{~m}^{2} \\
-40 \mathrm{~m}^{2}\end{array}$ & $\begin{array}{l}\text { buildings in neighbourhood } \\
\text { - mainly high-rise } \\
\text { - mixed low-rise and high-rise } \\
\text { - mainly low-rise }\end{array}$ \\
\hline monthly costs & depth backyard & green space \\
\hline - Nlg. 800 & -5 meters & - large central park \\
\hline - Nlg. 1100 & -10 meters & - a few fairly large public gardens \\
\hline - Nlg. 1400 & - 15 meters & - more small public gardens \\
\hline number of bedrooms & car park & shopping centre \\
\hline-2 & - central in neighbourhood & - outside district \\
\hline-3 & - in the street & - central (one big) \\
\hline-4 & - on private property & - in neighbourhood (a few small) \\
\hline
\end{tabular}

In the present study, a linear-additive utility function is assumed; thus, only main effects have to be estimated. To obtain unbiased estimates of the main effects, a fractional factorial design is chosen by which attribute levels are combined in such a way that the correlation structure between the attributes is orthogonal. A design involving the construction of 27 profiles was the smallest fractional design by which all main effects could be estimated. The 27 profiles are randomly placed in choice sets of three profiles each. The option "do not move" is added to each choice set as a base alternative. In order to avoid order effects, the placement of profiles in choice sets is randomized nine times.

Respondents were requested to choose in each choice set the housing alternative which they were most likely to move into. If none of the housing alternatives in the choice set were acceptable, they could choose the option "do not move", which served as a base option in each choice set. Respondents were requested to complete nine choice sets, which was the entire design in one of the nine different random orders.

\subsection{Sample}

Stated choice data were collected in early 1996. Respondents were selected from a previous survey, which was primarily intended to collect data on the housing situation and housing needs in the region. In this data file, those households were selected which indicated that they (i) are willing to move within five years, (ii) prefer new housing or have no preference regarding housing age, (iii) are looking for housing in Eindhoven, (iv) are willing to spend at least Nlg. 700 a month on housing, (v) prefer a house in a row, and (vi) agreed to participate in a choice experiment. A questionnaire containing the conjoint choice experiment was sent by mail to the selected households. A total of 184 respondents were contacted by mail, 
and 95 of them returned the form. This represents a response rate of almost 50 per cent.

As housing choice is often the result of a multi-person decision-making process, multi-person households were asked to complete the questionnaire in joint deliberation with all the household members which are involved in the housing choice. Our former research has found evidence for the hypothesis that models based on group tasks better predict group housing choices than models based on tasks completed by individual group members (Molin et al., 1995). In 62 per cent of the multi-person households, at least two persons, mostly husband and wife, completed the questionnaire together. Additionally, in about a quarter of the households with children age 14 or older, at least one child participated.

\subsection{Analysis and results}

The typical result of any stated choice experiment is a set of frequencies which indicates how often the various alternatives included in any particular choice set have been chosen. Because the experimental conditions that drive these choice sets were systematically varied, it is possible to derive unbiased estimates of the contribution of the experimentally varied attribute levels to preference and hence choice probabilities. These estimates can be obtained by decomposing the observed choice probabilities into the part-worth utilities that represent the utility of the attribute levels, given the assumed specification of the preference function and the assumed choice model. Thus, if a linear-additive preference function and a multinominal logit model have been assumed, the problem is to find the part-worth utilities that, given these assumptions, best reproduce the observed choice probabilities. To this end, the attribute levels are coded. Several options are available, but a useful coding scheme is effect coding. This implies that every attribute with say $\mathrm{L}$ levels, is coded in terms of $\mathrm{L}-1$ indicator variables. All but one attribute levels are coded 1 on the corresponding indicator variable; the remaining attribute level is coded -1 on all indicator variables. Consequently, the parameters corresponding with these indicator variables represent attribute level departures from consumer's average utility or preference.

Loglinear models, weighted/generalized least squares, and iteratively re-weighted least squares, to name a few, may all be used to estimate the parameters of the model, depending upon the specific statistical properties one wishes to achieve. In the present study, an iteratively re-weighted least squares algorithm which produces maximum likelihood estimates was used.

The estimation of the model results in a goodness-of-fit measure which indicates how well the estimated model is able to reproduce the observed choices in the experiment. Common goodness-of-fit measures are based on a comparison of the log-likelihood of the estimated model (L) with the log-likelihood of the zero model $\left(\mathrm{L}_{0}\right)$, the model in which the all parameters are assumed to be zero. In the present study, $\mathrm{L}$ is equal to -318.48 and $\mathrm{L}_{0}$ is equal to -600.96 . By these log-likelihoods, McFadden's Rho Square (the likelihood ratio index $=1-\mathrm{L} / \mathrm{L}_{0}$ ) is equal to .47 . 
McFadden's Rho Square has an interpretation comparable to the proportion of explained variance in linear modelling, but has typically lower values (though this depends on the level of aggregation). Taking this into account, we conclude that the estimated model reproduces the observed choices very well.

Table 1 summarizes the part-worth utility contributions of the attribute levels and their estimated standard error. A positive part-worth means that the presence of the attribute level in a housing alternative increases the total utility for that alternative. All estimated part-worth utility contributions are in the anticipated direction. Let us now summarize the most important results.

(i) Owner-occupied houses are preferred to rental houses.

(ii) Residential utility decreases with increasing monthly costs from 800 to 1400 guilders. This relationship is almost perfectly linear.

(iii) Utility increases with increasing number of rooms. The increase in utility between two and three bedrooms is much higher than between three and four bedrooms.

(iv) Utility increases by increasing the size of the living room.

(v) Utility increases in almost a perfectly linear way with depth of backyard from five to 15 meters.

(vi) Car park on private property is preferred to car park in the street. The latter, in turn, is preferred to car park at a central place in the neighbourhood.

(vii) A neighbourhood with mainly low-rise buildings is preferred. A neighbourhood with mixed low- and high-rise buildings is almost as negatively evaluated as a neighbourhood with mainly high-rise buildings.

(viii) None of the part-worth utility levels of concentration of green space are significant.

(ix) Utility increases with decreasing distance to a shopping centre.

As Table 1 indicates, the part-worth utilities differ substantially between the attributes. To quantify this, we calculate the attributes' relative importance by considering the attributes' ranges. An attribute's range is the difference between the highest and the lowest estimated part-worth utility of its levels. The ranges of all attributes are summed and the percentual contribution of each attribute to this sum is calculated. The resulting relative importances of the attributes are presented in Figure 2. This figure shows that monthly costs is by far the most important attribute, with a relative importance of more than 30 per cent. This is followed by the attributes size of living room, number of bedrooms, and tenure, with a relative importance of 15 per cent or slightly less. The attributes concerning the space around the house (buildings in the neighbourhood, the depth of the backyard, car park and distance to shopping centre) hardly reach a relative importance of 7.5 per cent each. The green space concentration clearly is the least important attribute.

Perhaps the most important advantage of stated choice models is that choice probabilities for new housing alternatives can be predicted without any ad hoc and untestable assumptions regarding the relationship between preference and choice. To illustrate this, we predict latent choices for residences in Meerhoven. Under the assumption that no other residences are available at the same time, we predict the 
Table 1 Estimated part-worth utility contributions and their standard errors

\begin{tabular}{|c|c|c|}
\hline & part-worth utility & standard error \\
\hline constant & -1.42 & $0.12 *$ \\
\hline \multicolumn{3}{|l|}{ tenure } \\
\hline - rent & -0.55 & $0.11 *$ \\
\hline - own & $(0.55)$ & \\
\hline \multicolumn{3}{|l|}{ monthly costs } \\
\hline - Nlg. 800 & 1.27 & $0.10 *$ \\
\hline - Nlg. 1100 & 0.02 & 0.10 \\
\hline - Nlg. 1400 & $(-1.29)$ & \\
\hline \multicolumn{3}{|l|}{ number of bedrooms } \\
\hline-2 & -0.71 & $0.09 *$ \\
\hline-3 & 0.30 & $0.08 *$ \\
\hline-4 & $(0.40)$ & \\
\hline \multicolumn{3}{|l|}{ size of living room } \\
\hline$-20 \mathrm{~m}^{2}$ & -0.65 & $0.14 *$ \\
\hline$-30 \mathrm{~m}^{2}$ & 0.12 & 0.10 \\
\hline$-40 \mathrm{~m}^{2}$ & $(0.52)$ & \\
\hline \multicolumn{3}{|l|}{ depth of backyard } \\
\hline-5 meters & -0.23 & $0.09 *$ \\
\hline - 10 meters & -0.06 & 0.10 \\
\hline-15 meters & $(0.29)$ & \\
\hline \multicolumn{3}{|l|}{ car park } \\
\hline - central in neighbourhood & -0.22 & $0.11 *$ \\
\hline - in the street & -0.03 & 0.14 \\
\hline - on private property & $(0.25)$ & \\
\hline \multicolumn{3}{|l|}{ buildings in neighbourhood } \\
\hline - mainly low-rise & 0.40 & $0.09 *$ \\
\hline - mixed low-rise and high-rise & -0.18 & 0.09 \\
\hline - mainly high-rise & $(-0.22)$ & \\
\hline \multicolumn{3}{|l|}{ green space } \\
\hline - large central park & 0.07 & 0.09 \\
\hline - a few fairly large public gardens & 0.02 & 0.09 \\
\hline - more small public gardens & $(-0.08)$ & \\
\hline \multicolumn{3}{|l|}{ shopping centre } \\
\hline - outside district & -0.22 & $0.09 *$ \\
\hline - central (one big) & 0.00 & 0.09 \\
\hline - in neighbourhood (a few small) & $(0.22)$ & \\
\hline
\end{tabular}

(1) Not estimated but derived: by definition utility contributions of levels belonging to one attribute sum to zero.

* $\quad$ significant at the alpha $=0.05$ level. 
Figure 2 Relative importance of attributes (in percentages)

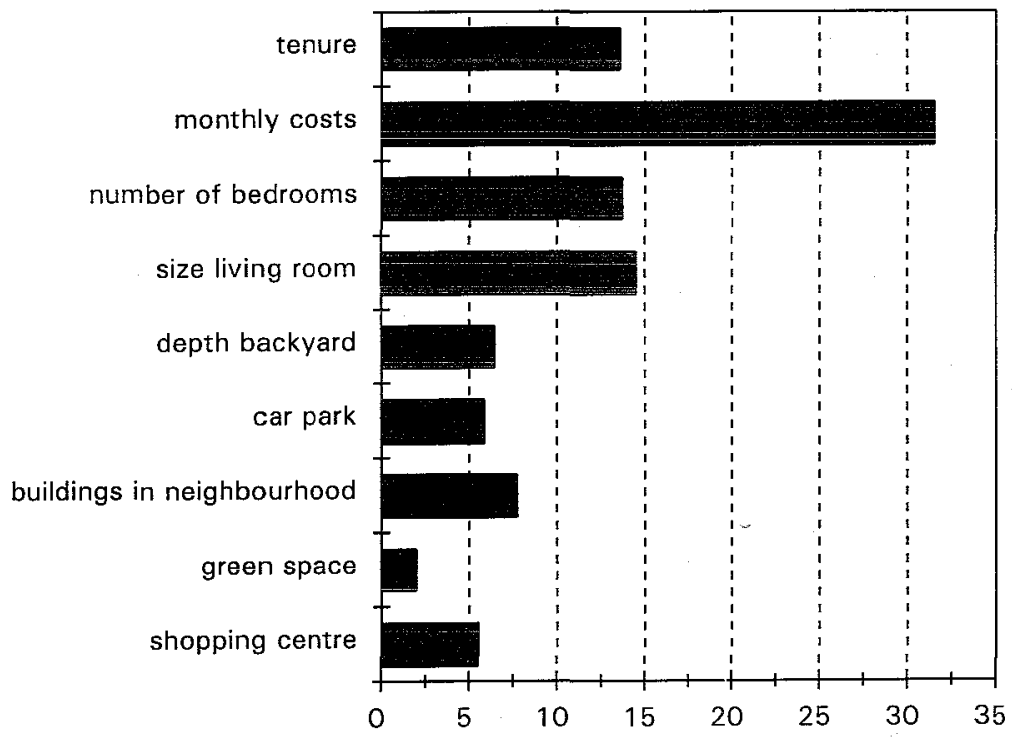

percentage of households that will choose a specified rental or owner-occupied house or none of either type. Moreover, we predict the choices for two different environments which are classified as a high- and a low-density neighbourhood.

Table 2 demonstrates that almost twice as many households choose an owneroccupied house compared to those choosing a rental house. This applies to both lowand high-density environments. Although an owner-occupied house is more expensive, its larger living room and owner-occupancy more than compensate for this. Comparing the low- and high-density environment makes it clear that a lowdensity environment is preferred. If both houses are offered in a low-density environment, 16.4 per cent of the households will not choose any of these houses and will not move. If the same houses are built in a high-density environment, this percentage rises to 41.9 per cent.

In the simulation described above, we implicitly assumed that the price remains constant across both environments. However, this assumption is not very realistic. Building land is a scarce resource, and its price will probably rise with decreasing density. Another question that arises is how much more the prospective occupants are willing to pay for lower-density housing. To answer this question, we examine how much the price in the low-density condition may rise until the choice probability for the houses in this condition is as low as the choice probability for the same houses in the high-density condition at low cost, as shown in Table 2. That point turns out to be an increase in monthly costs by approximately 300 guilders. Thus, we may conclude that prospective occupants are willing to pay 300 guilders more each month to live in low-density housing instead of high-density housing. 
Table 2 Latent choices for residential options in high- and low-density conditions (in percentages)

\begin{tabular}{rrrr} 
owner-occupied & rental & do not & total \\
3 bedrooms & 3 bedrooms & move \\
$40 \mathrm{~m}^{2}$ living room & $30 \mathrm{~m}^{2}$ living room & \\
central park & central park & \\
central shopping c. & central shopping c. & \\
Nlg. 1000 a month & Nlg. 800 a month & \\
\hline
\end{tabular}

LOW DENSITY

$12.5 \mathrm{~m}$ backyard

carpark on private

property

mainly low-rise buildings

55.3

28.4

16.4

100.0

HIGH DENSITY

$7.5 \mathrm{~m}$ backyard central car park mixed low-rise and high-rise buildings

\section{Conclusion and discussion}

The purpose of the present article was to discuss problems typical of eliciting housing preference. An attempt was made to explain some fundamental positions that have inspired different approaches to modelling consumer response in general and housing in particular. This discussion has led to the argument that among its rivals, stated preference and choice models have a few features that not only differentiate them from competing approaches but also make these models potentially powerful in eliciting consumer preference for housing. This is not to say that stated preference and choice models are necessarily error-free. Unlike other approaches, these models do allow one to test the validity of the assumptions one always has to make, either explicitly or implicitly, when measuring behaviour. The approach is illustrated in an example of new housing construction in Meerhoven.

The results of the study support the suggested approach, as all parameter estimates are in anticipated directions. Moreover, the choice model was quite capable of reproducing choices as derived from the experiment. The results of the model thus provide valuable information to policy-makers in terms of the relative importance of the attributes. It gives them guidelines as to which attributes are critical for their target market to accept new housing. New houses and residential areas can be designed to reflect these housing preferences.

The results of our application suggest that new row-houses in Meerhoven should be offered mainly as owner-occupied houses. As price is very important, these houses should not be too expensive and should contain at least three bedrooms and a $30-\mathrm{m}^{2}$ living room. Furthermore, choice probabilities for new row-houses increase, 
but to a lesser extent than the previously mentioned attributes, with increasing depth of backyard, decreasing distance to car park and shopping centre, and decreasing amount of high-rise buildings in the neighbourhood.

We also illustrated that latent choices can be predicted for residential options in a variety of possible conditions. In our simulation, it appeared that if two specified housing types are built in a high-density environment, far fewer households will choose to move than when the same houses are built in a low-density environment. If monetary values are attached to the attributes, assessments are possible of how much people are willing to pay for particular kinds of housing. In our study, we found that households are willing to pay approximately 300 guilders a month more for living in a low-density environment.

In sum, this project has provided additional evidence of the potential value of stated choice experiments in housing research. In the past, critics have argued that the approach is too complex, but the empirical evidence suggests this claim to be unwarranted, as long as the experiment is professionally designed and implemented. Moreover, we prefer a perhaps slightly less reliable, but more valid model to a very reliable, but invalid and hence useless instrument. Critics have aiso expressed scepticism about the assumption that stated preferences are systematically related to actual behaviour. Yet empirical evidence collected in other fields of application does not support this criticism. At least one could argue that stated preference models predict latent demand for housing. More importantly, because the external validity of these models in housing research has hardly been tested, the further advancement of the field is in need of studies that examine the external validity of stated choice experiments and that build conjoint-based simulation models of housing market clearing processes.

\section{References}

Louviere, J.J. (1988) Analysing decision making: metric conjoint analysis, Sage University Paper, Series on Quantitative Applications in Social Sciences, No. 07-067, Beverly Hills: Sage.

Louviere, J.J., and H.J.P. Timmermans (1990a) "A review of recent advances in decompositional preference and choice models", Tijdschrift voor Economische en Sociale Geografie (81), pp. 214-225.

Louviere, J.J., and H.J.P. Timmermans (1990b) "Hierarchical information integration applied to residential choice behavior", Geographical Analysis (22), pp. 127-145.

Molin, E.J.E., H. Oppewal, H.J.P. Timmermans (1995) "Modelling family decision making: a conjoint based approach", paper presented at the Association of Consumer Research Conference, Minneapolis, October.

Timmermans, H.J.P. (1984) "Decompositional multiattribute preferences models in spatial choice analysis", Progress in Human Geography (16), pp. 32-42. 
Timmermans, H.J.P. (1989) "Een decompositioneel hiërarchisch model voor woningkeuze: theorie en illustratie", in Musterd, S. (ed.), Methoden voor Woning en Woonmilieubehoeften Onderzoek, pp. 46-72, Amsterdam: SISWO.

Timmermans H., and L. van Noortwijk (1995) "Context dependencies in housing choice behavior", Environment and Planning A (27), pp. 181-192.

Tversky, A. (1972) "Elimination-by-Aspects: a theory of choice, Psychological Review (79), pp. 281-299.

\section{About the authors}

Eric Molin is Ph.D. candidate at the Department of Architecture and Urban Planning, Eindhoven University of Technology, Eindhoven, The Netherlands.

Harmen Oppewal is assistant professor at the Department of Architecture and Urban Planning, Eindhoven University of Technology, Eindhoven, The Netherlands.

Harry Timmermans is professor of Urban Planning at the Department of Architecture and Urban Planning, Eindhoven University of Technology, Eindhoven, The Netherlands. He also holds the Carthy Foundation Chair in Marketing at the Department of Marketing and Economic Analysis, University of Alberta, Edmonton, Canada. 